\title{
(IN) SEGURANÇA ALIMENTAR DE COMUNIDADES QUILOMBOLAS DO TOCANTINS
}

\author{
Estelamaris Tronco Monego ${ }^{1}$, Maria do Rosário Gondim Peixoto ${ }^{2}$, Mariana de Morais Cordeiro ${ }^{3}$, \\ Renata Medeiros Costa ${ }^{4}$
}

Este estudo buscou identificar a prevalência e alguns determinantes de (In) Segurança Alimentar (IA/SA) nas famílias de 14 comunidades quilombolas do estado do Tocantins (Brasil). Foram estudadas condições de moradia, prevalência de insegurança alimentar obtida pela Escala Brasileira de Insegurança Alimentar (EBIA) e participação em programas de alimentação e de transferência de renda. Foram estudadas $696(83,15 \%)$ famílias quilombolas, das $837(100 \%)$ identificadas. A IA esteve presente em 589 (85,1\%) famílias. Observou-se que $280(40,2 \%)$ domicílios eram de adobe, apenas 177 (25,4\%) possuíam coleta de lixo, 218 $(31,3 \%)$ dispunham de abastecimento de água e 59 (8,5\%) de esgotamento sanitário. Possuíam filtro de água e geladeira 455 $(65,6 \%)$ e $384(55,2 \%)$ famílias respectivamente. Receberam doação de alimentos 314 (45,8\%) famílias e 333 (47,8\%) não eram beneficiárias do Programa Bolsa Família (PBF). Apresentaram associação com IA a prática de "queimar ou enterrar o lixo", "ter abastecimento de água por carro pipa ou diretamente do rio ou córrego", "ter esgotamento a céu aberto", "não possuir geladeira" e "ser beneficiário do PBF". Conclui-se que a situação de insegurança alimentar é altamente prevalente nas comunidades quilombolas, que possuem também precárias condições de vida, sendo urgente a implementação de ações que garantam SA para as mesmas.

Palavras- chave: segurança alimentar, comunidade rural, vulnerabilidade social, quilombola

\section{FOOD INSECURITY OF TOCANTINS'S QUILOMBOLAS COMMUNITIES}

This study tried to identify the prevalence and some defining aspects of food (in) security (FS/FI) in the families of fourteen quilombola communities in the state of Tocantins( Brazil). It was studied living conditions, prevalence of food insecurity obtained from Brazilian Scale of Food Insecurity (EBIA) and participation in nutrition and transference income programs. It was studied 696 quilombola families (83,15\% from the total identified, which was 837 families). The FI was detected in $589(85,1 \%)$ families. It was observed that $280(40,2 \%)$ houses were made from adobe. Only $177(25,4 \%)$ had: garbage collection; $218(31,3 \%)$ water supply and 59 ( 8,5\%) sewage system. $455(65,6 \%)$ families had filtered water and $384(55,2 \%)$ refrigerator. Food donation was received by $314(45,8 \%)$ families and $333(47,8 \%)$ were Programa Bolsa Família's (PBF) beneficiary. Some practices were related to the FI such as: "burn or bury the trash", "getting water from pipas trucks or directly from the river or lake", "the swage be exposed", "not having refrigerator" and "be a beneficiary of the PBF". In conclusion, there's a clearly situation of food insecurity in the quilombola communities, therefore it's urgent the implementation of some actions in order to guarantee the food security of those families.

Key words: food security, rural community, social vulnerabilty, quilombola

\footnotetext{
${ }^{1}$ Faculdade de Nutrição - Universidade Federal de Goiás. Av. João Leite, 360 Casa 27. Setor Santa Genoveva. CEP. $74670-040$ Goiânia (GO) Brasil.Tel.55 (62) 3209-6270 Ramal 209. E-mail: emonego@fanut.ufg.br

2 Professora Adjunta da Faculdade de Nutrição / Universidade Federal de Goiás. E-mail: mrg.peixoto@uol.com.br

3 Nutricionista. Consultora Técnica do Centro Colaborador em Alimentação e Nutrição do Escolar - CECANE-UFG. Faculdade de Nutrição / Universidade Federal de Goiás. E-mail: marianamcordeiro@gmail.com

${ }^{4}$ Graduada em Nutrição pela Faculdade de Nutrição / Universidade Federal de Goiás. E-mail : renata.nutri@gmail.com
} 


\section{Introdução}

Comunidades quilombolas são grupos populacionais remanescentes de antigos quilombos, constituindo uma representação da resistência dos negros brasileiros. Estão localizados fisicamente em várias regiões do país, notadamente nas áreas rurais; apresentam um relativo grau de isolamento geográfico o que implica em desigualdades sociais e de saúde. Seus costumes, tradições, condição social, cultural e econômica peculiares, os distinguem de outros setores da coletividade nacional [1].

O reconhecimento legal destas comunidades foi estabelecido a partir da Constituição Brasileira (1988), que no Artigo 68 das Disposições Constitucionais Transitórias garante aos remanescentes das comunidades de quilombos o direito à propriedade de suas terras: "Aos remanescentes das comunidades dos quilombos que estejam ocupando suas terras é reconhecida a propriedade definitiva, devendo o Estado emitir-lhes os títulos respectivos" [2].

Somente a partir de 1995 os grupos populacionais quilombolas começaram a despertar maior atenção por parte do governo, sendo criado o primeiro documento solicitando a regularização dos territórios destas comunidades e a implementação de políticas públicas voltadas a atender às demandas desta população. O Diário Oficial da União (maio 2009) divulgou a Portaria $\mathrm{n}^{\circ}$ 43, onde a Fundação Cultural Palmares (FCP) registra e certifica 36 comunidades como remanescentes de quilombos. Este número, agregado ao anterior, totaliza 1.342 comunidades oficialmente certificadas [3].

Há poucos estudos realizados referentes às questões da saúde e qualidade de vida de comunidades quilombolas. A Chamada Nutricional Quilombola (2006) tem caráter pioneiro, uma vez que não existia no Brasil, até então, um diagnóstico nacional do perfil socioeconômico das famílias quilombolas, muito menos da situação nutricional das crianças quilombolas. Os resultados desta pesquisa evidenciam que $11,6 \%$ das crianças que vivem nas comunidades remanescentes de quilombos apresentam déficit de altura para a idade. Quando a escolaridade é verificada, $13,7 \%$ daquelas crianças de mães com menos de quatro anos de estudo estão desnutridas. Ainda segundo dados do governo federal, a condição econômica também é determinante. Entre as crianças que vivem em famílias da classe E $(57,5 \%$ das avaliadas), a desnutrição chega a $15,6 \%$; e cai para $5,6 \%$ no grupo que vive na classe $\mathrm{D}$, na qual estão $33,4 \%$ do total das pesquisadas [4].

Estas informações permitem supor que as comunidades estudadas apresentam elevado grau de insegurança alimentar: as comunidades quilombolas encontram-se em situação precária de vida, com péssimas condições de moradia e acesso a serviços de água e esgoto. Esta evidencia permite supor que não há, entre as comunidades quilombolas, garantia do Direito Humano à Alimentação Adequada ${ }^{[5]}$.

"A seguranca alimentar e nutricional consiste na realização do direito de todos ao acesso regular e permanente a alimentos de qualidade, em quantidade suficiente, sem comprometer o acesso a outras necessidades essenciais, tendo como base práticas alimentares saudáveis, que respeitem a diversidade cultural e que sejam social, econômica e ambientalmente sustentáveis." (Art. $3^{\circ}$ Lei 11.346, de 15 de setembro de 2006 p.1) ${ }^{[6]}$.

O Direito Humano à Alimentação Adequada (DHAA) está associado não só à garantia do acesso diário a alimentos em quantidade e qualidade suficiente para atender as necessidades nutricionais para a manutenção da saúde, como também está relacionada ao direito de acesso aos recursos e meios para produzir ou adquirir alimentos seguros e saudáveis que respeite os hábitos e a cultura inerente do ser humano [7].

No estudo de Silva et al.[1] foram identificados diversos fatores que caracterizam as comunidades quilombolas em insegurança alimentar: falta de posse de terra, ausência de uma renda monetária, o aumento de doenças, os fatores ambientais, a marginalidade e o analfabetismo.

A falta de infra-estrutura é outro fator relacionado à insegurança alimentar. Algumas comunidades não possuem energia elétrica para facilitar a produção de alimentos e manter uma boa qualidade alimentar ${ }^{[8]}$. 
Segundo Panigasse, et al. [5] uma forma de conhecer as condições sócio-econômicas e conseqüentemente a situação de vida de determinada população é a Escala Brasileira de Insegurança Alimentar, a qual permite avaliar a insegurança alimentar intrafamiliar por meio da percepção do entrevistado.

Tendo como pressuposto os dados coletados na Chamada Nutricional Quilombolas e diante da importância da realização de estudos que avaliem a situação de segurança alimentar nestas comunidades, bem como a necessidade de dispor de indicadores de vida e de saúde para este grupo social, este trabalho visa identificar e analisar alguns determinantes de (in)segurança alimentar nas famílias de 14 comunidades quilombolas do estado do Tocantins.

\section{Metodologia}

Os dados deste estudo fazem parte da primeira etapa da pesquisa "Educação alimentar e nutricional: ferramenta básica na construção da segurança alimentar e nutricional às comunidades quilombolas do estado de Tocantins", projeto aprovado e parcialmente financiado pelo Centro Colaborador de Alimentação e Nutrição CECAN e pela Coordenação Estadual de Alimentação e Nutrição do Estado do Tocantins e aprovado pelo Comitê de Ética em Pesquisa da Universidade Federal de Goiás (CoEP/UFG) sob o numero 042/2007. Na primeira etapa da pesquisa foi realizado $\mathrm{O}$ diagnóstico de Insegurança Alimentar e Nutricional das famílias remanescente de quilombos.

Trata-se de um estudo de corte transversal, desenvolvido no período de julho a dezembro de 2008. A população do estudo foi composta pelas 837 familias residentes em 14 comunidades de remanescentes de quilombos no estado do Tocantins (Quadro 1), participando do estudo $696(83,15 \%)$ famílias, uma vez que 141 $(16,85 \%)$ não responderam o questionário por motivos diversos (não foram encontradas no domicílio, não moravam mais na região ou o domicílio encontrava-se fechado).

A coleta de dados foi realizada por três entrevistadores previamente treinados com auxílio de cooperadores técnicos e monitores da Superintendência do Programa Estadual de Alimentação e Melhoria da Qualidade de Vida (PROVIDA), sendo o mesmo antecedido de um estudo piloto. Em cada domicilio era entrevistado apenas um indivíduo adulto, cujo papel do cotidiano familiar permitisse que o mesmo respondesse questões referentes a todos os membros da família.

Quadro 1 - Distribuição das comunidades quilombolas selecionadas, segundo número de famílias identificadas e estudadas. Tocantins, Brasil, 2008.

\begin{tabular}{|l|c|c|c|c|}
\hline \multicolumn{1}{|c|}{ Comunidade } & \multicolumn{2}{c|}{ Famílias identificadas } & \multicolumn{2}{c|}{ Famílias estudadas } \\
\% & \multicolumn{2}{c|}{ n } & \multicolumn{2}{c|}{ n } \\
\hline Barra da Aroeira & 71 & 14,5 & 68 & 9,8 \\
\hline Chapada da Natividade & 117 & 13,0 & 108 & 15,5 \\
\hline Cocalinho & 136 & 16,2 & 92 & 13,2 \\
\hline Comunidade de Malhadinha & 60 & 7,2 & 56 & 8,1 \\
\hline Córrego Fundo & 22 & 2,6 & 19 & 2,7 \\
\hline Distrito do Morro São João & 64 & 7,6 & 50 & 7,2 \\
\hline Kalunga do Mimoso & 170 & 20,3 & 143 & 20,5 \\
\hline Laginha & 18 & 2,2 & 17 & 2,4 \\
\hline Lagoa da Pedra & 39 & 4,7 & 27 & 3,9 \\
\hline Mumbuca & 33 & 3,9 & 25 & 3,6 \\
\hline Povoado do Prata & 47 & 5,6 & 42 & 6,0 \\
\hline Redenção & 20 & 2,4 & 20 & 2,9 \\
\hline São Joaquim & 34 & 4,1 & 23 & 3,3 \\
\hline São José & 6 & 0,7 & 6 & 0,9 \\
\hline Total & 837 & 100,0 & 696 & 100,0 \\
\hline
\end{tabular}


As informações relativas às variáveis do estudo foram obtidas com a aplicação de um questionário contendo perguntas pré-codificadas. O questionário contemplava os seguintes ítens: identificação da família, características da entrevista, caracterização dos moradores, variáveis relativas ao domicílio, alimentação (programa de alimentação, segurança alimentar, produção de alimentos no domicílio, consumo de alimentos por grupo, recordatório de 24 horas), saúde da criança, estilo de vida, medidas antropométricas e pressão arterial.

No presente trabalho foram estudadas as características da unidade domiciliar (tipo de domicílio, abastecimento de água, tipo de coleta do lixo, tipo de esgotamento sanitário, presença de filtro de água e geladeira no domicílio) e as informações sobre participação em programas de alimentação (recebimento de doação de alimentos nos últimos seis meses) e segurança alimentar para adulto responsável pela alimentação da família. Quanto ao recebimento de outro benefício, além da doação de alimentos, consideraram-se somente as famílias beneficiadas pelo programa de transferência de renda Bolsa Família.

Para medir o nível de insegurança alimentar foi utilizada a Escala Brasileira de Insegurança Alimentar (EBIA) [9]. A EBIA consta de 15 perguntas centrais fechadas, com resposta do tipo [sim] ou [não] para situação nos últimos três meses. Do conjunto de questões, sete são direcionadas apenas às famílias onde há moradores menores de 18 anos. Cada resposta afirmativa representa um ponto e a somatório dos pontos avalia a insuficiência alimentar em diferentes níveis de intensidade: segurança alimentar $=$ zero; insegurança leve $=1-5$ pontos (em famílias com indivíduos $<18$ anos) ou 1-3 pontos (naquelas sem este grupo); insegurança moderada $=6-10$ ou 4-6 e a pontuação de 11-15 ou 7-8 para insegurança alimentar grave.

Antes do início da entrevista era lido o Termo de Consentimento Livre e Esclarecido, para que fosse assinado pelo participante ou capturada uma impressão dactiloscópica, no caso do mesmo ser analfabeto. Só foram entrevistados aqueles que cumpriram a formalidade.

As análises estatísticas foram realizadas utilizando-se o programa Epi Info versão 3.5.1 (2008). As prevalências foram expressas em porcentagens considerando intervalo de confiança de 95\% (IC 95\%). Testes de associação pela distribuição do qui-quadrado, com nível de significância de 5\%, foram utilizados para avaliar a associação entre as variáveis estudadas e a insegurança alimentar.

\section{Resultados}

Foram estudadas 696 famílias $(83,15 \%)$ de 14 comunidades quilombolas do estado do Tocantins. Destas, $679(81,12 \%)$ responderam o questionário na sua totalidade e $17(2,03 \%)$ apenas parte do mesmo. Como alguns itens do questionário não foram respondidos por todas as famílias, há variação do número total (n) de respostas em algumas variáveis.

A Tabela 1 apresenta as informações sobre a segurança alimentar (SA), presente em apenas $103(14,9 \%)$ famílias quilombolas. Das 589 $(85,1 \%)$ familias que se encontravam em situação de insegurança alimentar (IA), 258 (37,3\%) apresentaram IA leve, 228 (32,9\%) moderada e $103(14,9 \%)$ grave (Tabela 1$)$.

Tabela 1. Distribuição das freqüências e percentuais referentes à variável (in)segurança alimentar. Comunidades quilombolas, Tocantins, Brasil, 2008.

\begin{tabular}{lccc}
\hline Variável & $\mathbf{n}=\mathbf{6 9 2}$ & $\mathbf{\%}$ & IC 95\% \\
\hline Insegurança Alimentar & $\mathbf{5 8 9}$ & $\mathbf{8 5 , 1}$ & $\mathbf{8 2 , 2}-\mathbf{8 7 , 6}$ \\
$\quad$ Leve & 258 & 37,3 & $33,7-41,0$ \\
Moderada & 228 & 32,9 & $29,5-36,6$ \\
Grave & 103 & 14,9 & $12,4-17,8$ \\
& & & $\mathbf{1 2 , 4 - 1 7 , 8}$ \\
Segurança Alimentar & $\mathbf{1 0 3}$ & $\mathbf{1 4 , 9}$ & \\
\hline
\end{tabular}


As condições de moradia e saneamento básico (Tabela 2) encontradas indicaram que a maioria ( $\mathrm{n}=280 ; 40,2 \%$ ) das famílias quilombolas possuía casa do tipo adobe e apenas $77(11,1 \%)$ de alvenaria com acabamento completo. Apenas 177 $(25,4 \%)$ famílias tinham a coleta de lixo realizada por serviço de limpeza, sendo que a informação sobre o destino do lixo para $362(52,0 \%)$ famílias é queimar ou enterrar. A maioria das famílias
( $\mathrm{n}=478 ; 68,7 \%$ ) não possuía abastecimento de água por rede pública, sendo que 151 (21,7\%) famílias utilizavam água de rio ou córrego. A forma mais comum de esgotamento sanitário encontrada foi a céu aberto, presente em 327 $(47,0 \%)$ famílias. Mais da metade $(n=455 ; 65,6 \%)$ das famílias possuía filtro de água em seu domicilio, enquanto que $384(55,2 \%)$ domicílios possuíam geladeira.

Tabela 2. Distribuição das freqüências e percentuais referentes às condições de moradia e saneamento básico. Tocantins, Brasil, 2008.

\begin{tabular}{lccc}
\hline Variáveis & $\mathbf{n}$ & $\mathbf{\%}$ & IC 95\% \\
\hline Tipo de domicílio (n=696) & 280 & 40,2 & $36,6-44,0$ \\
Adobe & 77 & 11,1 & $8,9-13,7$ \\
Alvenaria com acabamento completo & 148 & 21,3 & $18,3-24,5$ \\
Alvenaria com acabamento incompleto & 191 & 27,4 & $24,2-30,9$ \\
Outros & & & \\
Tipo de coleta de lixo (n=696) & 177 & 25,4 & $22,3-28,9$ \\
Coletado por serviço de limpeza & 157 & 22,6 & $19,5-25,9$ \\
Colocado em céu aberto & 362 & 52,0 & $48,2-55,8$ \\
Queimado ou enterrado & & & \\
Tipo de abastecimento de água (n=696) & 41 & 5,9 & $4,3-8,0$ \\
Carro pipa & 174 & 25,0 & $21,9-28,4$ \\
Encanada* & 151 & 21,7 & $18,7-25,0$ \\
Pega no rio ou córrego & 87 & 12,5 & $10,2-15,2$ \\
Poço ou nascente & 218 & 31,3 & $27,9-34,9$ \\
Rede pública & 25 & 3,6 & $2,4-5,3$ \\
Outros & & & \\
Esgotamento sanitário (n=695) & 327 & 47,0 & $43,3-50,8$ \\
Céu aberto & 89 & 12,8 & $10,5-15,6$ \\
Fossa rudimentar & 209 & 30,1 & $26,7-33,7$ \\
Fossa séptica & 59 & 8,5 & $6,6-10,9$ \\
Rede pública & 11 & 1,6 & $0,8-2,9$ \\
Outro & & & \\
Possui filtro de água (n=694) & 228 & 32,8 & $29,4-36,5$ \\
Não possui filtro nem trata água & 11 & 1,6 & $0,8-2,9$ \\
Não possui filtro, mas trata a água** & 455 & 65,6 & $61,9-69,1$ \\
Possui filtro & & & \\
Possui geladeira (n=696) & 384 & 55,2 & $51,4-58,9$ \\
Sim & 312 & 44,8 & $41,1-48,6$ \\
Não & & \\
\hline & & & \\
& & &
\end{tabular}

* Encanada da serra, poço artesiano, rio ou córrego; **Fervida ou clorada

A investigação sobre benefícios sociais ou transferência de renda evidenciou que a maioria das familias ( $\mathrm{n}=371 ; 54,2 \%$ ) não recebeu doação de alimentos nos últimos seis meses a contar da data da entrevista, e que $363(52,2 \%)$ não estão cadastradas como beneficiárias do Programa Bolsa Familia (Tabela 3). 
Tabela 3. Distribuição das freqüências e percentuais referentes à doação de alimentos e cobertura do Programa Bolsa Família. Tocantins, Brasil, 2008.

\begin{tabular}{lccc}
\hline Variáveis & $\mathbf{n}$ & $\mathbf{\%}$ & IC 95\% \\
\hline $\begin{array}{l}\text { Recebimento de doação de alimento nos últimos } \mathbf{6} \\
\text { meses (n=685) }\end{array}$ & & & \\
Sim & 314 & 45,8 & $42,1-49,7$ \\
Não & 371 & 54,2 & $50,3-57,9$ \\
Beneficiário do Programa Bolsa Família (n=696) & & & \\
Sim & 333 & 47,8 & $44,1-51,6$ \\
Não & 363 & 52,2 & $48,4-55,9$
\end{tabular}

Para análise da IA em relação às características da unidade domiciliar verificou-se que não houve associação significante $(p>0,05)$ com as variáveis "tipo de domicílio" e "possui filtro de água” (Tabela 4).

Ainda na Tabela 4 pode-se observar que as variáveis relacionadas com saneamento básico, como "tipo de coleta de lixo", "tipo de abastecimento de água" e "esgotamento sanitário" tiveram associação estatisticamente significante $(p<0,05)$ com IA. As famílias que queimavam ou enterravam o lixo apresentaram maior porcentagem de IA ( $\mathrm{n}=320 ; 88,6 \%$ ).

As famílias com abastecimento por água encanada e aquelas com ligação à rede pública, $143(82,7 \%)$ e 174 (80,6\%), respectivamente, apresentaram menor insegurança alimentar do que aquelas privadas desse tipo de abastecimento. Outro dado verificado é que a IA foi mais presente naquelas familias que obtinham a água por meio do carro pipa $(\mathrm{n}=38 ; 92,7 \%)$ ou da coleta no rio ou córrego ( $\mathrm{n}=140 ; 92,7 \%)$. Nos domićlios em que o esgoto era a céu aberto encontrou-se maior porcentagem de IA ( $\mathrm{n}=289 ; 88,7 \%)$, enquanto que nos domicilios que dispunham de fossa séptica a porcentagem foi menor $(\mathrm{n}=165$; $79,3 \%)$.

A variável "possui geladeira" mostrou associação significante com a IA. Das 311 familias que não tinham geladeira no domicílio, $279(89,7 \%)$ apresentaram IA. 
Tabela 4. Análise da associação da insegurança alimentar com as condições de moradia e saneamento básico. Tocantins, Brasil, 2008.

\begin{tabular}{|c|c|c|c|}
\hline \multirow[b]{2}{*}{ Variáveis } & \multicolumn{3}{|c|}{ Insegurança Alimentar } \\
\hline & $\mathbf{n}$ & $(\%)$ & Valor de $p^{*}$ \\
\hline Tipo de domicílio $(\mathrm{n}=692)$ & & & 0,4154 \\
\hline Adobe & 245 & 87,8 & \\
\hline Alvenaria com acabamento completo & 64 & 84,2 & \\
\hline Alvenaria com acabamento incompleto & 124 & 83,8 & \\
\hline Outros & 156 & 82,5 & \\
\hline Tipo de coleta de lixo $(n=692)$ & & & $\mathbf{0 , 0 2 3 9}$ \\
\hline Coletado por serviço de limpeza & 143 & 81,7 & \\
\hline Colocado em céu aberto & 126 & 80,8 & \\
\hline Queimado ou enterrado & 320 & 88,6 & \\
\hline Tipo de abastecimento de água $(n=692)$ & & & $\mathbf{0 , 0 2 1 9}$ \\
\hline Carro pipa & 38 & 92,7 & \\
\hline Encanada $* *$ & 143 & 82,7 & \\
\hline Pega no rio ou córrego & 140 & 92,7 & \\
\hline Poço ou nascente & 74 & 85,1 & \\
\hline Rede pública & 174 & 80,6 & \\
\hline Outros & 20 & 83,3 & \\
\hline Esgotamento sanitário $(n=691)$ & & & $\mathbf{0 , 0 3 7 2}$ \\
\hline Céu aberto & 289 & 88,7 & \\
\hline Fossa rudimentar & 76 & 86,4 & \\
\hline Fossa séptica & 165 & 79,3 & \\
\hline Rede pública & 50 & 86,2 & \\
\hline Outros & 8 & 72,7 & \\
\hline Possui filtro de água $(n=690)$ & & & 0,1558 \\
\hline Não possui filtro nem trata água & 201 & 88,5 & \\
\hline Não possui filtro, mas trata a água*** & 10 & 90,9 & \\
\hline Possui filtro & 376 & 83,2 & \\
\hline Possui geladeira $(n=692)$ & & & $\mathbf{0 , 0 0 2 1}$ \\
\hline Sim & 310 & 81,4 & \\
\hline Não & 279 & 89,7 & \\
\hline
\end{tabular}

* Teste qui-quadrado; ** Encanada da serra, poço artesiano, rio ou córrego

*** Fervida ou clorada

Em relação à doação de alimentos, não houve associação estatisticamente significante com a IA $(p>0,05)$. O mesmo não ocorreu com as familias que eram beneficiadas pelo programa governamental Bolsa Família uma vez que 302 $(91,0 \%)$ destas apresentaram IA $(p<0,05)$ (Tabela 5). 
Tabela 5. Análise da associação da insegurança alimentar com doação de alimentos e cobertura do Programa Bolsa Família. Tocantins, Brasil, 2008.

\begin{tabular}{lccc}
\hline Variáveis & \multicolumn{3}{c}{ Insegurança alimentar } \\
\cline { 2 - 4 } & $\mathbf{N}$ & $\mathbf{( \% )}$ & Valor de $^{*}$ \\
\hline $\begin{array}{l}\text { Recebimento de doação de alimento nos últimos } \\
\text { 6 meses (n=682) }\end{array}$ & & & $\mathbf{0 , 0 7 0 7}$ \\
$\quad$ Sim & 275 & 87,6 & \\
Não & 304 & 82,6 & \\
Beneficiário do Programa Bolsa Família (=692) & & & $<\mathbf{0 , 0 0 0 1}$ \\
Sim & 302 & 91,0 & \\
Não & 287 & 79,7 & \\
\hline
\end{tabular}

\section{Discussão}

A constatação da grande ocorrência de IA $(85,1 \%)$ e das precárias condições dos domicílios demonstram a vulnerabilidade social e biológica a qual estão submetidas as comunidades quilombolas do Tocantins.

Frequência elevada de IA também foi observada por Favaro et al. ${ }^{[10]}$ em aldeias indígenas no Mato Grosso do Sul $(75,5 \%)$ e por Pereira et al. [11] no Jardim Jaqueline, região de alta vulnerabilidade social, em São Paulo (88,0\%). Estas altas prevalências de IA resultam de condições semelhantes às encontradas, tais como isolamento geográfico e/ou social, dificuldades de acesso a bens e serviço, concentração da terra, insuficiência de renda e precarização da educação.

Quanto à classificação das famílias segundo a presença de insegurança alimentar, observou-se que a IA leve e moderada, estavam presentes, respectivamente, em $37,3 \%$ e $32,9 \%$ das famílias. Fatores relacionados com a qualidade da alimentação e a preocupação com a falta do alimento em um futuro próximo estão presentes na IA leve, enquanto que na insegurança moderada começa a haver restrição quantitativa na alimentação dos adultos da família. Já a IA grave, presente em 14,9\% das famílias estudadas caracteriza-se por deficiência quantitativa de alimento à mesa, implicando na sensação de fome entre os adultos e crianças da família ${ }^{[12]}$.

De acordo com a Pesquisa Nacional por Amostra de Domićlilio - PNAD realizada em 2004 [13], a Região Norte do Brasil foi a que apresentou maior insegurança alimentar (46,3\%), ocorrência esta observada também no estado do Tocantins (46,7\%). Ao comparar os dados das comunidades quilombolas com os da PNAD, observa-se que a prevalência de IA (leve, moderada e grave) nas comunidades quilombolas do estado de Tocantins foi cerca de duas vezes maior do que aquelas encontradas nas demais regiões do país.

No que diz respeito ao tipo de domićlio, as familias quilombolas entrevistadas moravam em sua maioria em casas de adobe. Este resultado está de acordo com outro estudo realizado na comunidade Kalunga no estado de Goiás, onde boa parte das casas dessas famílias era feita com materiais encontrados na região, como adobe e palha [14]. Observou-se, no entanto, no presente estudo, que não houve associação significante entre $\mathrm{o}$ tipo de domicílio e a IA, ao contrário do estudo de Panigassi et al. [5] realizado com famílias residentes na zona urbana do município de Campinas (SP) que mostra associação estatisticamente significante entre a IA e o tipo precário de moradia (alvenaria inacabada, madeira, zinco ou papelão).

A falta ou precariedade de saneamento básico esteve presente nas comunidades pesquisadas remanescentes de quilombo de Tocantins. As informações sobre a coleta de lixo por serviço de limpeza $(25,4 \%)$, o abastecimento de água (31,3\%) e o esgotamento sanitário por rede pública $(8,5 \%)$ evidenciam que estes benefícios atingem uma pequena parcela dessa população. A precariedade de saneamento básico foi também observada no estudo nacional realizado em 2006 junto a 60 comunidades quilombolas rurais, com somente $1 / 3$ das residências estudadas ligadas à rede pública de esgoto ou dispunham de fossa séptica, além de estarem também ligadas a rede pública de água [15].

Silva et al. [1] em seu estudo com comunidades quilombolas do Pará observou que das seis comunidades estudadas, apenas uma contava com água encanada, porém sem nenhum tratamento sanitário. A inadequação 
das condições de saneamento, principalmente as relacionadas aos dejetos sanitários, foi apontada como um fator de aumento de risco de morbidades nos moradores das comunidades quilombolas, em função da contaminação dos rios durante os períodos de enchente [16].

Ao relacionar as variáveis referentes ao saneamento básico (tipo de abastecimento de água, coleta de lixo e esgotamento sanitário) com IA, observou-se associação significante entre elas. De uma forma geral, um dos menores índices de insegurança alimentar foi encontrado nas comunidades que dispunham de saneamento básico disponibilizado pela rede pública.

A falta de acesso ao saneamento básico pode comprometer a utilização dos alimentos pelo organismo, seja pela contaminação microbiológica ou mesmo física destes, comprometendo a segurança alimentar do indivíduo [17]. Pesquisa realizada com cinco mil titulares do cartão Bolsa Família, em 229 municípios brasileiros distribuídos nas cinco regiões brasileiras, mostrou que a falta de acesso a bens públicos básicos, tais como o esgoto, interferem diretamente nas taxas de IA [18]. Já no estudo de Salles-Costa et al. [19] realizado em uma amostra de base populacional do Rio de Janeiro não mostrou associação entre as condições de saneamento básico com a segurança alimentar, porém esse resultado foi justificado em função das características gerais desse serviço na região do estudo, não permitindo a discriminação entre as famílias.

A presença de filtro de água não teve associação com a IA neste estudo, porém deve-se ressaltar a evidência que são muitas as famílias que não faziam nenhum tratamento da água $(32,8 \%)$. Salles-Costa et al. [19] estudando a prevalência de IA nas famílias residentes em um dos distritos mais pobres do município de Duque de Caxias (RJ), verificaram que a presença de filtro para tratamento da água apresentou associação significante com a IA, sendo esta maior para aquelas famílias que não tratavam a água.

$\mathrm{Na}$ zona rural, o risco de contaminação por doenças infecciosas e diarréicas causadas pelo consumo de água não potável é grande. Estudos alegam que um determinante do déficit de crescimento de crianças são as doenças causadas pelo consumo de água não potável, o que reforça a importância do tratamento da água para o consumo [20,21].

Quase metade das famílias entrevistadas não possuía geladeira no domicílio. Houve associação significante entre a ausência de geladeira e a IA, e 89,7\% das famílias sem geladeira apresentavam IA. Não foram encontrados estudos que apresentassem a associação entre a SA e a presença de geladeira no domicilio, mas Menegolla et al. [21] concluíram que a falta de geladeira nos domicílios de famílias indígenas da Região do Sul do Brasil colocava em risco o crescimento linear das crianças devido ao efeito sobre a conservação dos alimentos.

Em relação à cobertura do Programa Bolsa Familia (PBF), observou-se que esse benefício era recebido por $333(47,8 \%)$ famílias entrevistadas. Essa informação não difere muito do resultado obtido no inquérito realizado em 2006 com 60 comunidades quilombolas na "Chamada Nutricional de Crianças Quilombolas Menores de Cinco Anos de Idade" onde $51,7 \%$ dos entrevistados eram beneficiários desse programa de transferência de renda [15]. Já no estudo com comunidades quilombolas no município de Santarém (PA), observou-se que apenas 3,3\% das famílias entrevistadas tinham como fonte de renda esse programa ${ }^{[1]}$.

Um estudo detalhado da associação entre o benefício do PBF e a IA permitiu verificar que entre aqueles que recebem esse benefício a IA foi maior, porém não foi questionado aos participantes do estudo há quanto tempo eram beneficiários desse programa. Pesquisa realizada pelo IBASE [13] mostrou que, apesar do aumento na quantidade e na variedade dos alimentos proporcionados pelo benefício do $\mathrm{PBF}$, a situação de IA é alta, sendo que a maior parte do público atendido por esse programa (83\%) se enquadra num dos três níveis em que se classifica a IA: grave, moderada e leve. Sendo assim, concluiu-se que o PBF é importante para melhorar as condições de vida das familias, porém por si só não garante índices satisfatórios de segurança alimentar.

De uma forma geral, observa-se que a IA entre as comunidades quilombolas estudadas tende a diminuir à medida que as condições de moradia e acesso aos bens e serviços melhoram.

Diante do exposto, é lícito afirmar que as políticas públicas voltadas à garantia da segurança alimentar devem extrapolar o Setor Saúde, buscando uma perspectiva intersetorial, abrangendo a capacidade aquisitiva da população e a escolha dos alimentos que devem ser consumidos, levando ainda em consideração os fatores culturais que interferem nessa seleção [22].

\section{Conclusões}

O direito humano à alimentação adequada constitui-se em um dos direitos fundamentais da 
humanidade. Este direito não está sendo garantido às famílias quilombolas do estado do Tocantins, que foram objeto deste estudo.

A insegurança alimentar esteve fortemente presente. Um dos aspectos estudados que mostrou maior relevância foi o relato sobre a (falta de) qualidade da alimentação e preocupação com sua obtenção. A restrição e/ou deficiência quantitativa na alimentação e até mesmo a fome esteve presente em uma parcela significativa das famílias quilombolas.

Os resultados mostram que são precárias as condições de moradia das comunidades quilombolas estudadas. Dentre as variáveis estudadas, queimar ou enterrar o lixo, ter abastecimento de água por carro pipa ou por meio da coleta em rio ou córrego, possuir esgotamento sanitário a céu aberto, não possuir geladeira e ser beneficiário do Programa Bolsa Família foram os fatores que mostraram maior associação estatística com a insegurança alimentar.

Conclui-se que as dificuldades enfrentadas pelas famílias quilombolas são muitas e apontam para a necessidade de implementar ações conjuntas dos serviços de saúde pública com outros setores estruturantes da qualidade de vida das populações.

É preciso que o Estado brasileiro em parceria com a comunidade científica, amplie os estudos, buscando encontrar rumos que encaminhem para o empoderamento destas comunidades. Dentre os caminhos a serem trilhados sugere-se a ampliação da produção de alimentos para o auto-consumo; a busca de alternativas para a obtenção de acesso ao saneamento básico; a formação de educadores sintonizados com a fixação da criança quilombola em seu território e a utilização de ferramentas da promoção da saúde junto aos diferentes segmentos da comunidade, com vistas a oportunizar autonomia e melhoria na qualidade de vida destas populações.

\section{Agradecimentos}

Por terem participado na construção intelectual do conjunto deste estudo, agradecemos a Ilian Delvia Vasconcelos C. de Souza (PROVIDA - TO), Terezinha de Jesus P. Franco (Secretaria de Saúde do estado do Tocantins) e Maria de Fátima Gil (FANUT/UFG).

\section{Referências Bibliográficas}

1. SILVA DO, GUERRERO AFH, GUERREO CH, TOLEDO LM. A rede de causalidade da insegurança alimentar e nutricional de comunidades quilombolas com a construção da rodovia BR-163, Pará, Brasil. Revista de Nutrição. 2008; 21: 83-97.

2. BRASIL. Constituição (1988). Constituição da República Federativa do Brasil. Brasília/DF 1988.

3. Ministério da Cultura. Fundação Cultural Palmares. Mais 36 comunidades quilombolas são certificadas no país, 2009. [Texto eletrônico] 2009 [citado em 2009 set 17]. Disponível em: http://www.palmares.gov.br.

4. Ministério do Desenvolvimento Social e Combate à Fome. Políticas Sociais e Chamada Nutricional Quilombola: estudos sobre condições de vida nas comunidades e situação nutricional das crianças. Cadernos de Estudos Desenvolvimento Social em Debate. Brasília; 2008. BRASIL. 142 p.

5. PANIGASSI G, SEGALL-CORREAA AM, MARINLEÓN L, PÉREZ-ESCAMILLA R, SAMPAIO MEA, MARANHA LK. Insegurança alimentar como indicador de iniqüidade: análise de inquérito populacional. Caderno de Saúde Pública. 2008; 24 (10): $2376-2384$.

6. Diário Oficial da União. Lei $\mathrm{n}^{\circ}$ 11.346, de 15 de setembro de 2006. Cria o Sistema Nacional de Segurança Alimentar e Nutricional - SISAN com vistas em assegurar o direito humano à alimentação adequada e dá outras providências. Brasília; 2006. BRASIL.

7. VALENTE FLS. Direito Humano à alimentação: desafios e conquistas. São Paulo: Cortez; 2002.

8. CHEQUER J. Segurança alimentar na balança. Portal do IBASE [texto eletrônico] 2005 [citado em 2009 jan 20]. Disponível em: www.ibase.br.

9. SEGALL-CORRÊA ERP, SAMPAIO MFA, MARINLEON L, PANIGASSI G, MARANHA LK. et. al. (In) Segurança Alimentar no Brasil. Validação de metodologia para acompanhamento e avaliação. Relatório Técnico, Campinas SP; 2004.

10. FAVARO T, RIBAS DLB, ZORZATTO JR, SEGALL-CORREAA AM, PANIGASSI G. Segurança alimentar em famílias indígenas Terena, Mato Grosso do Sul, Brasil. Caderno de Saúde Pública. 2007; 23 (4): $785-793$.

11. PEREIRA DA, VIEIRA VL, FIORE EG, CERVATO-MANCUSO, AM. Insegurança alimentar em região de alta vulnerabilidade social da cidade de São Paulo. Segurança Alimentar e Nutricional. 2006; 13 (2): $34-42$.

12. SEGALL-CORRÊA AM. Insegurança alimentar medida a partir da percepção das pessoas. Estudos Avançados. 2007; 21 (60): 143 - 154. 
13. Fundação Instituto Brasileiro de Geografia e Estatística (IBGE). Pesquisa Nacional por Amostra de Domicílios 2004: Segurança Alimentar. Rio de Janeiro; 2006.

14. NEIVA ACGR, SERENO JRB, SANTOS AS, FIORAVANTI MCS. Caracterização socioeconômica e cultural da comunidade quilombola Kalunga de Cavalcante, Goiás, Brasil: dados preliminares. In: IX Simpósio Nacional Cerrado. II Simpósio Internacional Savanas Tropicais. ParlaMundi, Brasília: 2008.

15. [15] Ministério do Desenvolvimento Social e Combate à Fome. Chamada Nutricional Quilombola, 2006. Resumo Executivo. Brasília: 2007. BRASIL.

16. [16] GUERRERO AFH, SILVA DO, TOLEDO LM, GUERRERO JCH, TEIXEIRA P. Mortalidade Infantil em Remanescentes de Quilombos do Município de Santarém - Pará, Brasil. Revista Saúde e Sociedade. 2007; 16 (2): 103-110.

17. [17] CARVALHO MC. Segurança Alimentar. Revista e Portal SESC São Paulo [periódico eletrônico] 2009 [citado em 2009 set 10]; (141). Disponível em: http://www.sescsp.org.br/sesc/revistas.

18. Instituto Brasileiro de Análises Sociais e Econômicas (IBASE). Documento síntese: Repercussões do Programa Bolsa Família na Segurança Alimentar e Nutricional das Famílias Beneficiadas, 2008, p1-20.
19. SALLES-COSTA R, PEREIRA RA, VASCONCELLOS MTL, VEIGA GV, MARINS VMR, JARDIM BC, et al. Associação entre fatores socioeconômicos e insegurança alimentar: estudo de base populacional na Região Metropolitana do Rio de Janeiro, Brasil. Revista de Nutrição. 2008; 21: 99 - 109.

20. AMARAL LA, NADER FILHO A, ROSSI JUNIOR OD, FERREIRA FLA, BARROS LSS. Água de consumo humano como fator de risco à saúde em propriedades rurais. Revista de Saúde Pública. 2003; 37 (4): $510-514$.

21. MENEGOLLA IA, DRACHLER ML, RODRIGUES IH, SCHWINGEL LR, SCAPINELLO E, PEDROSO $\mathrm{MB}$, et al. Estado nutricional e fatores associados à estatura de crianças da Terra Indígena Guarita, Sul do Brasil. Caderno de Saúde Pública. 2006; 22 (2): 395 406.

22. Ministério da Saúde. Secretaria de Atenção à Saúde. Departamento de Atenção Básica. Política Nacional de Alimentação e Nutrição. Brasília/DF 2003. 48 p. 